\title{
Analysis of Important Gene Ontology Terms and Biological Pathways Related to Pancreatic Cancer
}

\author{
Hang Yin, ${ }^{1}$ ShaoPeng Wang, ${ }^{2}$ Yu-Hang Zhang, ${ }^{3}$ Yu-Dong Cai, ${ }^{2}$ and Hailin Liu ${ }^{1}$ \\ ${ }^{1}$ Department of Gastroenterology, Ninth People’s Hospital, School of Medicine, Shanghai Jiao Tong University, Shanghai 200011, China \\ ${ }^{2}$ School of Life Sciences, Shanghai University, Shanghai 200444, China \\ ${ }^{3}$ Institute of Health Sciences, Shanghai Institutes for Biological Sciences, Chinese Academy of Sciences, Shanghai 200031, China
}

Correspondence should be addressed to Hailin Liu; liuhailin@medmail.com.cn

Received 31 May 2016; Revised 18 July 2016; Accepted 7 September 2016

Academic Editor: Yungang Xu

Copyright (c) 2016 Hang Yin et al. This is an open access article distributed under the Creative Commons Attribution License, which permits unrestricted use, distribution, and reproduction in any medium, provided the original work is properly cited.

\begin{abstract}
Pancreatic cancer is a serious disease that results in more than thirty thousand deaths around the world per year. To design effective treatments, many investigators have devoted themselves to the study of biological processes and mechanisms underlying this disease. However, it is far from complete. In this study, we tried to extract important gene ontology (GO) terms and KEGG pathways for pancreatic cancer by adopting some existing computational methods. Genes that have been validated to be related to pancreatic cancer and have not been validated were represented by features derived from GO terms and KEGG pathways using the enrichment theory. A popular feature selection method, minimum redundancy maximum relevance, was employed to analyze these features and extract important GO terms and KEGG pathways. An extensive analysis of the obtained GO terms and KEGG pathways was provided to confirm the correlations between them and pancreatic cancer.
\end{abstract}

\section{Introduction}

Pancreatic cancer has been widely reported as a malignant tumor subtype involving one of the most significant tissue organs that contribute to both digestive system and endocrine system, the pancreas. Based on clinical symptoms and genetic characteristics, pancreatic cancer can be clustered into various subtypes [1]. Among such subtypes, pancreatic ductal adenocarcinoma (PDAC) accounts for more than $90 \%$ of all the cases. With a specific low survival rate (18\% for one-year survival rate and $5 \%$ for five-year survival rate), pancreatic cancer results in more than thirty thousand deaths around the world and has been regarded as one of the top killers for human beings $[1,2]$.

Although pancreatic cancer has been included in the list of top killers for human beings, the biological processes and mechanisms that contribute to the initiation and progression of pancreatic cancer have not been fully revealed. Based on recent publications, the underlying mechanisms of pancreatic cancer have been partially uncovered mainly by experimental trials $[3,4]$. The traditional experimental trials that contribute to revealing of pancreatic cancer associated genes and pathways can be divided into two levels: the nucleotide level (DNA/RNA) and the protein level. At the nucleotide level, polymerase chain reaction (PCR), highthroughput sequencing, and gene chips (either genomic chips or expression profile chips) contribute to the identification of the genomic and transcriptional background for pancreatic cancer initiation and progression [5]. Taking gene chip as an example, such experimental tool reveals the detailed genetic and expression profile characteristics of tumor cells and has been reported to contribute to the identification of various pancreatic cancer associated biological processes, including DPC4 tumor-suppressor pathway and the famous MAPK signaling pathway which we will analyze below [68]. As for the protein level, western blot turns out to be the most commonly used biochemical method to identify the expression and activation status of a known protein in certain in vivo or in vitro environment. Further, relying on in vitro gene expression (RNA) interference technologies, the characteristic alteration of the expression and function of a series of proteins that have been identified on such two levels as we have mentioned above can be validated and such group of proteins can be further concluded into various biological 
processes and pathways $[9,10]$. Based on the experimental technologies we have mentioned above, various principal regulatory pathways have been identified and confirmed to contribute to the initiation and progression of pancreatic cancer.

Based on existing publications, various principle regulatory pathways and biological processes that contribute to the initiation and progression of pancreatic cancer have been identified. Such signaling pathways and biological processes contribute to three main aspects of the biological processes of pancreatic cancer: transmembrane signal transduction, intracellular metabolic transduction, and the intranuclear proliferative regulation $[3,11,12]$. Different signaling pathways have been identified to contribute to different biological processes of pancreatic cancer during tumorigenesis. According to recent literatures, ErbB signaling pathway and TGF-beta signaling pathway participate in the transmembrane signal transduction of pancreatic cancer $[13,14]$. Such transmembrane signal transduction pathways have been further validated to transfer the signals to intracellular pathways (such as p53 signaling pathway, MAPK signaling pathway, PI3K-Akt signaling pathway, and VEGF signaling pathway) [13, 15-17]. Intracellular signaling pathways have been identified to contribute to the abnormal proliferation of pancreatic cells and further initiate the tumorigenesis. Taking MAPK signaling pathway as an example, as the downstream region of Ras signaling pathway, MAPK signaling pathway contributes to the phosphorylation of two crucial families of proteins, ERK and JNK, and further regulates proliferative signaling transportation into the nucleus [8]. Although various functional pathways have been revealed to contribute to the abnormal proliferation during the tumorigenesis of pancreatic cancer, the core trigger for the initiation of pancreatic cancer turns out to be the abnormal intranuclear proliferative regulation [18]. It has been identified that two main biological processes contribute to the abnormal proliferation of pancreatic cells during tumorigenesis: the inhibition of cell apoptosis and the excessive activation of proliferation [19]. All of such regulatory signaling pathways have been reported to be abnormal in pancreatic cancer and further contribute to the tumorigenesis. However, according to such signaling pathways, we still cannot explain all the pathological phenotypes of pancreatic cancer, implying that there are still core regulatory pathways remaining to be uncovered.

The study on the underlying mechanism of pancreatic cancer has lasted for decades [20]. However, based on experimental methods, only limited genes and pathways are proved to contribute to pancreatic cancer. The experimental methods that contribute to the identification and confirmation of pancreatic cancer associated pathways are quite expensive and time-consuming. Recently, with the development of computational biology and bioinformatics, various computational methods have been presented to predict cancer, including pancreatic cancer associated genes [21]. However, up to now, few computational methods have been present to describe the detailed functional pathways and biological processes of pancreatic cancer. In computational biology, KEGG pathways and gene ontology (GO) terms are widely used to describe the detailed and specific biological processes in human cells. KEGG (Kyoto Encyclopedia of Genes and Genomes) has been widely regarded as an integrated database resource for gene and protein annotation [22]. Based on KEGG database, we can obtain the KEGG pathway maps which reflect the functional pathway based network in living cells [22]. On the other hand, GO is a bioinformatics initiative to unify the presentation of gene and gene product attributes across all species [23]. Therefore, KEGG pathways and GO terms can provide a more accurate and clearer panorama for the underlying biological processes of pancreatic cancer.

In this study, we applied a popular feature selection method, minimum redundancy maximum relevance (mRMR) [24], to extract a group of pancreatic cancer associated KEGG pathways and GO terms, filling the gaps of current study in pancreatic cancer. First, genes that have been validated to be related to pancreatic cancer were deemed positive samples, while other genes were deemed negative samples. Second, the enrichment theory of GO term and KEGG pathway was adopted to encode each gene. Third, all GO terms and pathways were analyzed by mRMR method and some of the important ones were extracted. Finally, the extracted GO terms and KEGG pathways were extensively discussed to confirm their relationships to pancreatic cancer.

\section{Materials and Methods}

2.1. Materials. The validated genes related to pancreatic cancer were retrieved from the KEGG pathway, which is a main database in KEGG database [25]. 65 validated genes were extracted from the pathway hsa05212 (http://www. genome.jp/kegg-bin/show_pathway? map=hsa05212\&show description=show, accessed in December 2014). These genes were termed as positive samples, comprising the gene set $S_{p}$, and are listed in Supplementary Material I available online at http://dx.doi.org/10.1155/2016/7861274. To extract the GO terms and KEGG pathways that are specific to pancreatic cancer, it is necessary to employ some genes that are not related to pancreatic cancer. Since we used the enrichment scores of GO terms and KEGG pathways to indicate the associations between genes and GO terms (KEGG pathways), genes without these scores were not considered in this study. Up to now, there are 18,600 genes whose GO and KEGG enrichment scores can be calculated. Beside the 65 genes related to pancreatic cancer, each of the remaining 18,535 genes can be deemed a negative sample because the probability of it being related to pancreatic cancer is not very high. These 18,535 genes comprised the gene set $S_{n}$. The whole gene set $S$ was constructed by combining the genes in $S_{p}$ and $S_{n}$; that is, $S=S_{p} \cup S_{n}$.

2.2. Feature Construction. To extract important GO terms and KEGG pathways that are related to pancreatic cancer, it is necessary to encode each gene in $S$ based on all GO terms and KEGG pathways. Here, we used the enrichment theory of GO term and KEGG pathway to encode each gene, which can indicate the relationships between genes and GO terms (KEGG pathways). Then, the difference between positive and 
negative samples can be distinguished by the key features produced by a feature selection method, which would be described in Section 2.3. The encoding procedure is shown as follows.

GO Enrichment Score. The GO enrichment score was utilized to represent the quantitative correlation between each GO term and involved genes. For a given $\mathrm{GO}$ term $\mathrm{GO}_{j}$ and a gene $g$, let $G_{1}$ be a gene set consisting of genes annotated to $\mathrm{GO}_{j}$ and $G_{2}$ be another gene set consisting of neighbor genes of $g$ in the protein-protein interaction network reported in STRING (http://string-db.org/) [26], a well-known public database providing known and predicted protein-protein interactions. The $\mathrm{GO}$ enrichment score between $\mathrm{GO}_{j}$ and $g$ is defined as the $-\log _{10}$ of the hypergeometric test $P$ value [2730] of $G_{1}$ and $G_{2}$, which can be calculated by

$$
\mathrm{ES}_{\mathrm{GO}}\left(g, \mathrm{GO}_{j}\right)=-\log _{10}\left(\sum_{k=m}^{n} \frac{\left(\begin{array}{c}
M \\
k
\end{array}\right)\left(\begin{array}{c}
N-M \\
n-k
\end{array}\right)}{\left(\begin{array}{c}
N \\
n
\end{array}\right)}\right),
$$

where $N$ is the number of genes in human, $M$ is the number of genes in $G_{1}, n$ is the number of genes in $G_{2}$, and $m$ is the number of common genes of $G_{1}$ and $G_{2}$. A large enrichment score between $\mathrm{GO}_{j}$ and $g$ indicates close relationship between them. In this study, we considered 12,511 GO terms, inducing 12,511 GO enrichment scores for each gene, which can be obtained by an in-house program using $\mathrm{R}$ function phyper. The $\mathrm{R}$ code is "score $\leftarrow-\log 10$ (phyper(numWdrawn - 1, numW, numB, numDrawn, lower.tail=FALSE))," where numW, numB, and numDrawn are the number of genes annotated to $\mathrm{GO}_{j}$, the number of genes not annotated to $\mathrm{GO}_{j}$, and the number of neighbors of gene $g$ and numWdrawn is the number of neighbors of gene $g$ that are also annotated to $\mathrm{GO}_{j}$

KEGG Enrichment Score. Similar to that of the GO terms, the relationship between KEGG pathways and genes in $S$ can be represented by the KEGG enrichment scores. For a given KEGG pathway $K_{j}$ and a gene $g$, let $G_{1}$ be a gene set consisting of genes in $K_{j}$ and $G_{2}$ is same as $G_{2}$ in the above paragraph. The KEGG enrichment score between $K_{j}$ and $g$ is also defined to be the $-\log _{10}$ of the hypergeometric test $P$ value [29-31] of $G_{1}$ and $G_{2}$, which can be computed by

$$
\mathrm{ES}_{\mathrm{KEGG}}\left(g, K_{j}\right)=-\log _{10}\left(\sum_{k=m}^{n} \frac{\left(\begin{array}{c}
M \\
k
\end{array}\right)\left(\begin{array}{c}
N-M \\
n-k
\end{array}\right)}{\left(\begin{array}{c}
N \\
n
\end{array}\right)}\right),
$$

where the definitions of $N, M, n$, and $m$ are same as those in (1). Also, a high score between a KEGG pathway $K_{j}$ and a gene $g$ indicates they have strong associations. Here, we considered 239 KEGG pathways, resulting in 239 KEGG enrichment scores for each gene, which can also be obtained by an inhouse program using $\mathrm{R}$ function phyper.

As mentioned above, each gene $g$ was represented by 12,511 features derived from GO terms and 239 features derived from KEGG pathways, which can be formulated as a vector

$$
\begin{gathered}
f(g)=\left(\mathrm{ES}_{\mathrm{GO}}\left(g, \mathrm{GO}_{1}\right), \ldots, \mathrm{ES}_{\mathrm{GO}}\left(g, \mathrm{GO}_{12511}\right),\right. \\
\left.\mathrm{ES}_{\mathrm{KEGG}}\left(g, K_{1}\right), \ldots, \mathrm{ES}_{\mathrm{KEGG}}\left(g, K_{239}\right)\right)^{T} .
\end{gathered}
$$

2.3. Feature Selection Method. As described in Section 2.2, each gene was represented by 12,750 features derived from the GO terms and KEGG pathways. Considering the unequal roles of these features on pancreatic cancer, that is, some features playing more important roles than others, it is necessary to employ some advanced tools to analyze them, thereby extracting key features that are strongly associated with pancreatic cancer. Here, a reliable and widely used feature selection method, namely, mRMR method [24], was adopted to analyze all investigated 12,750 features. The mRMR method was proposed by Peng et al. [24] and was deemed to be a useful tool to analyze the feature space of complicated problems. Up to now, it has been widely applied to analyze various complicated biological systems or problems [32-45].

The mRMR method has two excellent criteria: MaxRelevance and Min-Redundancy. The criterion of MaxRelevance measures the importance of features based on their correlation to targets, while the criterion of Min-Redundancy gives a guarantee that the selected features have minimum redundancies. It is clear that the former criterion can be used to extract important features for a classification problem, while if one tries to construct an optimal feature subspace, two of them should be used. Because the purpose of this study is to extract key features that are closely related to the pancreatic cancer but not to construct an optimal feature subspace, we only used the Max-Relevance in this study. For each feature, let $f$ be a variable representing values of all samples under the feature and $c$ be the target variable. The mutual information (MI) value of each feature can be computed by

$$
I(c, f)=\iint p(c, f) \log \frac{p(c, f)}{p(c) p(f)} d c d f,
$$

where $p(c)$ and $p(f)$ are the marginal probabilities of $c$ and $f ; p(c, f)$ is the joint probabilistic distribution of $c$ and $f$. In fact, MI measures the mutual dependence between two variables. Furthermore, it has wide applications because it can deal with random variables that are not real-valued. Thus, mRMR method adopted MI to measure the relevance of each feature. According to the MI values of all features, a feature list, namely, MaxRel feature list, can be built. The MaxRel feature list was formulated as

$$
F_{M}=\left[f_{1}^{M}, f_{2}^{M}, \ldots, f_{N}^{M}\right]
$$

where $N$ represented the total number of features. Clearly, features with high ranks in this list are more likely to be related to pancreatic cancer. Extensive investigation of the corresponding GO terms and KEGG pathways may give new insights for the study of pancreatic cancer. 


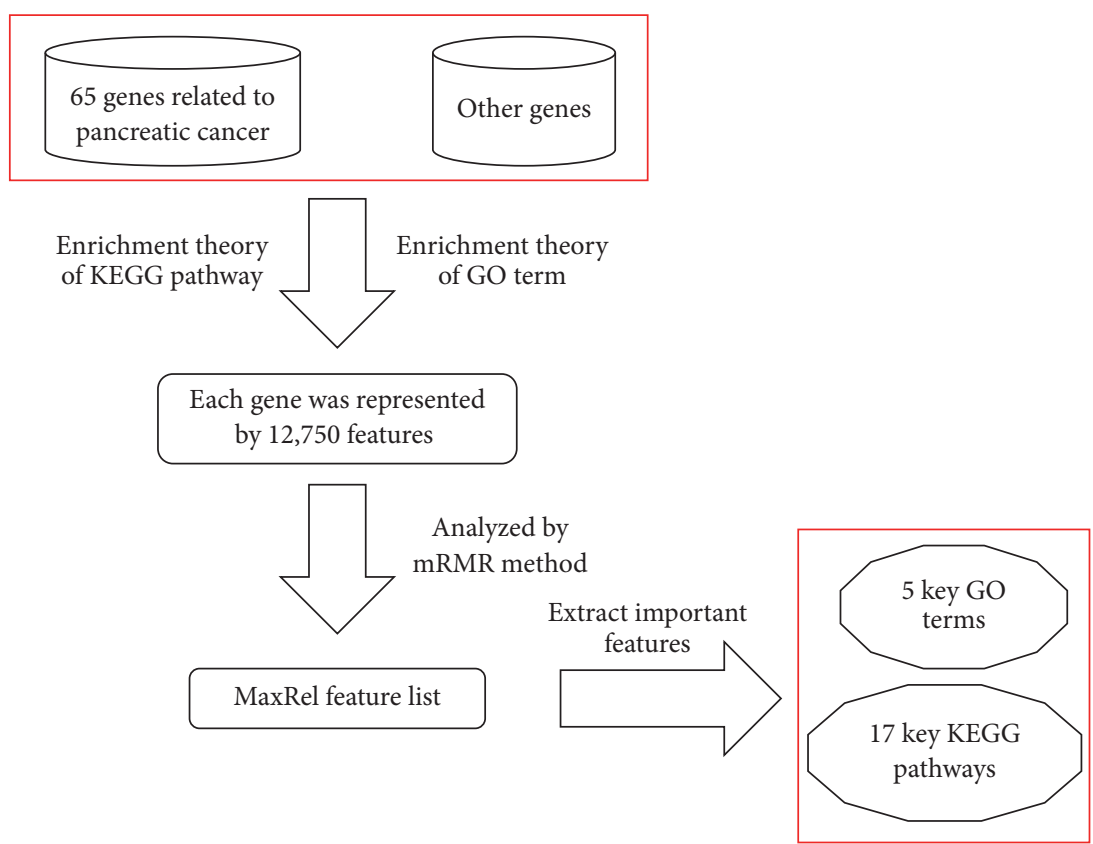

FIGURE 1: The procedures for extracting important KEGG pathways and GO terms of pancreatic cancer.

\section{Results and Discussion}

The purpose of this study is to extract important KEGG pathways and GO terms of pancreatic cancer using some computational methods. The detailed procedures are illustrated in Figure 1.

3.1. Results. As described in Section 2.2, each gene in $S$ was represented by 12,750 features derived from the GO terms and KEGG pathways. These features were analyzed by $\mathrm{mRMR}$ method described in Section 2.3 by calculating their relevance to targets measured by their MI values. According to the MI value of each feature, the MaxRel feature list was constructed, which is provided in Supplementary Material II.

It is known that not all GO terms and KEGG pathways have strong associations with pancreatic cancer. The rank of a corresponding feature in the MaxRel feature list for a GO term or a KEGG pathway indicates its importance for pancreatic cancer. Thus, we can select the GO terms and KEGG pathways whose features received high ranks in the MaxRel feature list to investigate their importance. Here, we chose the 22 features receiving MI values no less than 0.01 for further analysis, resulting in 22 GO terms or KEGG pathways. Their detailed information is listed in Tables 1 and 2. It can be observed from these two tables that there are 17 important KEGG pathways (listed in Table 1) and five key GO terms (listed in Table 2). In the following section, detailed discussion on these GO terms and KEGG pathways would be given.

3.2. Analysis of Key KEGG Pathways and GO Terms. As shown in Tables 1 and 2, 17 KEGG pathways and five GO terms were extracted, which are deemed to be highly related to pancreatic cancer. According to recent published literature, all of these KEGG pathways and GO terms identified in this study have been confirmed to participate in pancreatic cancer associated biological processes.

3.2.1. KEGG Pathways Associated with Pancreatic Cancer. 17 KEGG pathways were extracted in this study, which are deemed to be associated with the initiation and progression of pancreatic cancer.

(1) Pathways Describe Various Tumor Subtypes. Among the 17 KEGG pathways, 10 KEGG pathways describe the whole metabolic regulatory network of a specific cancer subtype. KEGG pathway hsa05200 describes the kernel regulatory factors that contribute to the initiation and progression of pan-cancer. Various pathways (e.g., Wnt signaling pathway, cAMP signaling pathway, and VEGF signaling pathway) in such network (hsa05200) and functional genes (e.g., PKA, $R h o$, and $V E G F$ ) have been identified in pancreatic cancer [46-48]. Taking gene PKA and its corresponding signaling pathway, the cAMP signaling pathway, as an example, cyclic AMP associated pathway and PKA have been identified and confirmed to contribute to the migration and invasion of pancreatic cancer, validating our prediction [48].

Apart from the KEGG pathways describing the pancancer, various KEGG pathways have also been predicted to describe the detailed subtypes of cancer. Among them, hsa05223 which describes the regulatory network and pathways of non-small-cell lung cancer has been predicted to be related to the specific biological processes of pancreatic cancer. Such KEGG pathway contains various tumor associated factors and pathways (such as KRAS, TP53, and functional pathways that they participate in). It has been proved that KRAS and TP53 as we have mentioned above have both been reported and confirmed to contribute to the initiation and 
TABLE 1: 17 important KEGG pathways for pancreatic cancer.

\begin{tabular}{lccc}
\hline KEGG pathway ID & KEGG pathway & MI value & Rank in MaxRel feature list \\
\hline hsa05211 & Renal cell carcinoma & 0.011 & 1 \\
hsa04010 & MAPK signaling pathway & 0.011 & 3 \\
hsa05212 & Pancreatic cancer & 0.011 & 4 \\
hsa05200 & Pathways in cancer & 0.011 & 5 \\
hsa05210 & Colorectal cancer & 0.011 & 6 \\
hsa05214 & Glioma & 0.011 & 7 \\
hsa05220 & Chronic myeloid leukemia & 0.011 & 8 \\
hsa05223 & Non-small-cell lung cancer & 0.01 & 10 \\
hsa04510 & Focal adhesion & 0.01 & 11 \\
hsa05213 & Endometrial cancer & 0.01 & 12 \\
hsa05221 & Acute myeloid leukemia & 0.01 & 13 \\
hsa05215 & Prostate cancer & 0.01 & 14 \\
hsa05160 & Hepatitis C & 0.01 & 16 \\
hsa04012 & ErbB signaling pathway & 0.01 & 20 \\
hsa04660 & T cell receptor signaling pathway & \\
hsa04150 & mTOR signaling pathway & 0.01 & \\
hsa04722 & Neurotrophin signaling pathway & 0.01 & \\
\hline
\end{tabular}

TABLE 2: Five important GO terms for pancreatic cancer.

\begin{tabular}{lccc}
\hline GO term ID & GO term & MI value & Rank in MaxRel feature list \\
\hline GO: 0007265 & Ras protein signal transduction & 0.011 & 2 \\
GO: 0048011 & Neurotrophin-TRK receptor signaling pathway & 0.01 & 15 \\
GO: 0016772 & Transferase activity, transferring phosphorus-containing groups & 0.01 & 17 \\
GO: 0016303 & 1-Phosphatidylinositol-3-kinase activity & 0.01 & 19 \\
GO: 0004713 & Protein tyrosine kinase activity & 0.01 & 21 \\
\hline
\end{tabular}

progression of pancreatic cancer $[49,50]$. Considering factors like KRAS and TP53 which have been identified in both pancreatic cancer associated pathways and non-small-cell lung cancer associated pathways, such two regulatory networks (pancreatic cancer and non-small-cell lung cancer associated pathways) may definitely interact with each other, and our predicted KEGG term hsa05223 may actually participate in pancreatic associated pathways validating the accuracy and efficacy of our prediction. Apart from non-small-cell lung cancer, another four subtypes of cancer (prostate cancer, endometrial cancer, renal cell carcinoma, and colorectal cancer) associated biological processes have also been predicted to be associated with pancreatic cancer. There are various core regulatory factors and pathways in prostate cancer associated pathways ( $h$ sa05215). Steroid hormone biosynthesis has been reported to contribute to the metastasis of prostate cancer and interacts with the specific oncogene of pancreatic cancer $A R$, implying its core role for the prostate associated pathways that we have predicted $[51,52]$. According to recent publications, such core pathway of the steroid hormone biosynthesis may also contribute to pancreatic cancer, revealing the underlying relationships between prostate cancer associated pathways (as we have predicted, hsa05215) and pancreatic cancer [53, 54].

Pathways of endometrial cancer ( $h s a 05213)$, a malignant neoplasm involving the female genital system, have also been predicted to be associated with pancreatic cancer. The core regulatory factors of endometrial cancer and pancreatic cancer have quite a lot of crosstalk and overlap. Take $\beta$-catenin as an example, $\beta$-catenin has been revealed to participate in the Wnt signaling pathway in various tumor subtypes $[55,56]$. The initiation and progression of both pancreatic cancer and endometrial cancer have been confirmed to be associated with Wnt signaling pathway, implying that such two regulatory networks may have crosstalk and our extracted KEGG pathway (hsa 05213) may actually contribute to the progression of pancreatic cancer [57-59]. As for the other three important solid tumor associated pathways, two of them, renal cell carcinoma and colorectal cancer associated pathways ( $h s a 05211$ and $h s a 05210$ ), contain functional regulatory genes and pathways that have been reported to contribute to pancreatic cancer at the same time. In renal cell carcinoma, $M E T$ has been reported to interact with the hepatocyte growth factor (HGF) and turns out be the initial signal for $M A P K$ signaling pathway [60]. Coincidentally, in pancreatic cancer, $M E T$ has also been confirmed to be quite a crucial gene for tumor initiation, progression, and metastasis, implying the crosstalk of pathways associated pancreatic cancer and renal cell carcinoma [61, 62]. As for colorectal cancer associated pathways (hsa05211), during the tumorigenesis of colorectal cancer, chromosomal instability (CIN) has been revealed to be a core driver mechanism and pathogenesis of the initiation [63]. In pancreatic cancer, CIN has also 
been regarded as a common phenotype and pathogenic factor, implying the undergoing relationship between such two regulatory networks [64]. According to Table 1, we also obtained the specific KEGG pathway describing pancreatic cancer associated pathway ( $h s a 05212$ ) which is definitely associated with pancreatic cancer, validating the accuracy and efficacy of our prediction.

Apart from such solid tumor subtype, two leukemia subtypes and sarcoma associated pathways have also been obtained to contribute to the tumorigenesis of pancreatic cancer. KEGG pathway, hsa05220, describes pathogenic biological processes of chronic myeloid leukemia (CML). Various factors have been reported to contribute to the chronic myeloid leukemia. PI3K-AKT pathway has been identified as a core component of chronic myeloid leukemia associated pathways as we have predicted [65]. Based on recent publications, such pathway (PI3K-AKT pathway) has also been confirmed to be quite crucial for pancreatic cancer, validating our prediction [66]. Apart from that, the specific $B C R-A B L$ fusion gene has also been identified in some pancreatic cancer patients, implying that $B C R-A B L$ fusion gene may also contribute to the tumorigenesis of pancreatic cancer [67]. Apart from CML associated pathways, regulatory networks that contribute to another nonsolid tumor subtype, acute myeloid leukemia (AML) (hsa05221), have also been contained in our results. As we all know, KRAS, STAT, and their respective regulatory pathways have all been associated with our predicted pathway (hsa05221) [68, 69]. As we have mentioned above, KRAS has been identified as a core regulatory factor that contributes to pancreatic cancer [49]. According to recent publications, STAT as AML associated gene has also been reported to contribute to pancreatic cancer, validating our prediction of pancreatic cancer associated genes [70]. Glioma, rising from glial cells, is a malignant sarcoma involving the brain and central nervous system. Based on our results, glioma associated pathways (hsa05214) may also contribute to pancreatic cancer. Genes associated with glioma such as EGFR (as oncogene) and PTEN (as tumor suppressor) have also been reported to contribute to the initiation and progression of pancreatic cancer $[71,72]$.

(2) Detailed Pathways That May Participate in Tumorigenesis. Apart from pathways that directly describe the tumorigenesis, four KEGG pathways that describe the detailed pathways were also extracted. KEGG pathway, hsa04150, which describes the mTOR signaling pathway has been predicted to contribute to pancreatic cancer. The relationship between mTOR signaling pathway and pancreatic cancer has been revealed by multiple recent publications [73-75]. As a regulatory mechanism for cell proliferation, mTOR signaling pathway has been confirmed to have crosstalk with various core regulatory factors and their respective signaling pathways including MAPK, TP53, RAS, and EGFR [76-79]. Some of such regulatory factors have also been contained in our results. MAPK signaling pathway (hsa04010) has been confirmed to have crosstalk with mTOR signaling pathway as we have mentioned above and has been reported to be quite crucial for the invasion and metastasis of pancreatic cancer [80, 81]. Apart from such two functional signaling pathways, another pathway, which has been widely reported to contribute to endometrial cancer, $E R B B$ signaling pathway (hsa04012), is also in Table 1 [82]. During the initiation and progression of pancreatic cancer, $E R B B$ signaling pathway has been confirmed to participate in the biological processes, validating our newly presented algorithm [83]. Neurotrophins have firstly been identified as a group of proteins that contribute to the survival, development, and function of neurons [84]. However, recent publications have revealed that neurotrophins may participate in the survival and proliferation of various cell types including the tumor cells [85-87]. Such functional protein, neurotrophin, which is regulated by another functional gene TRK has also been reported to contribute to pancreatic cancer, validating the efficacy of our prediction [88]. In Table 1, a specific KEGG pathway, hsa04722, which describes the neurotrophin signaling pathway was also listed. Based on our analyses, such biological process may definitely contribute to pancreatic cancer.

(3) Specific Pathways That Contribute to Cell-Cell Interaction. The last three KEGG pathways with MI values no less than 0.01 have been confirmed to contribute to the cell-cell/cellprotein interaction associated pathways. KEGG pathway hsa04510 describes the focal adhesion associated pathways. The abnormal activation of focal adhesion associated pathway has been widely reported in pancreatic cancer, implying that focal adhesion may be core biological processes during the tumorigenesis of pancreatic cancer [89]. Apart from focal adhesion, another biological process involving cell-cell interaction, $\mathrm{T}$ cell receptor signaling pathway (hsa04660), was also extracted in this study. It has been widely reported that the $\mathrm{T}$ cell receptor signaling pathway has been blocked or abnormally regulated in tumor microenvironment [90]. In pancreatic cancer, the initiation and progression of pancreatic cancer also interfere with the normal function of $\mathrm{T}$ cell receptor. Considering the recognition and cytolysis functions of $\mathrm{T}$ cells, the tumor cells and the $\mathrm{T}$ cells may put the selective pressure on each other and coevolve [91]. During the evolutionary processes, $\mathrm{T}$ cells with high recognition and cytolysis ability, which are both induced by $\mathrm{T}$ cell receptor signaling pathway, are all screened out, leaving dysfunctional $\mathrm{T}$ cells in tumor microenvironment $[92,93]$. Such coevolution processes imply the regulatory role of $\mathrm{T}$ cell receptor signaling pathway in pancreatic cancer. We also obtained a functional signaling pathway (hsa05160) that is related with the infection of hepatitis $C$ virus. Based on metaanalysis and case-control study, the infection of specific virus (hepatitis B and hepatitis C) has been confirmed to increase the risk of pancreatic cancer, validating the prediction based on our new algorithm, though the undergoing mechanism has not been fully revealed $[94,95]$.

3.2.2. GO Terms Associated with Pancreatic Cancer. Apart from the KEGG pathways mentioned above, five GO terms (listed in Table 2) that describe different biological processes were also extracted in this study, which are also deemed to contribute to the tumorigenesis of pancreatic cancer. The detailed analyses are listed below. 
GO: 0048011, which describes the neurotrophin-TRK receptor signaling pathway, has been predicted to contribute to pancreatic cancer. As we have mentioned above, neurotrophin-TRK receptor has been reported to contribute to the growth and progression of human pancreatic cancer [96]. Such evidence validates the efficacy and accuracy of our prediction algorithm. Apart from that, another GO term (GO: 0016772) describes the transferase activity, especially for the activity of transferring phosphorus-containing groups. During the progression of pancreatic cancer, transferases, especially for those that contribute to the transferring phosphorus-containing groups, have been identified to contain various variants and function abnormally. Take a classical transferase $S p h K 1$ as an example, SphK1 as a tumor associated protein has been reported to be overexpressed in pancreatic cancer [97]. Recent publications have confirmed that SphK1 regulates the sphingolipid metabolism and further contributes to the resistance against gemcitabine, a widely used anticancer drug for pancreatic cancer, validating the underlying role of phosphorus associated transferase for pancreatic cancer [97]. Another GO term (GO: 0016303) describes the specific activity of 1-phosphatidylinositol-3kinase $(P I 3 K)$. As we have mentioned above, $P I 3 K$ associated pathway has been widely reported to contribute to pancreatic cancer [66]. Similarly, the remaining two GO terms (GO: 0004713 and GO: 0007265) describe the protein tyrosine kinase $(P T K)$ activity and the Ras protein signal transduction, respectively, which have also been reported to contribute to pancreatic cancer. The inhibitors for protein tyrosine kinase have been widely used in clinical treatment for pancreatic cancer, implying the driving effect of PTK for pancreatic cancer [98]. As for Ras protein signal transduction, proteins of Ras family have been widely reported to contribute to tumorigenesis [3, 99]. A specific protein of Ras family, $K-R A S$, has been confirmed to be a driver gene for pancreatic cancer, validating the accuracy of our prediction.

According to the analyses listed above, all extracted functional KEGG pathways and GO terms are confirmed to definitely contribute to pancreatic cancer. Some new findings may give new insights for the study of pancreatic cancer or other types of cancer.

\section{Conclusions}

In this study, effective features, derived from the GO terms and KEGG pathways, were utilized to encode the genes related to pancreatic cancer. After being analyzed by the mRMR method, 22 key features were extracted, corresponding to five GO terms and 17 KEGG pathways. These GO terms and KEGG pathways may be the novel materials to investigate pancreatic cancer. Furthermore, they may also be useful to build an effective computational method for identification of novel genes related to pancreatic cancer. In future, we will try our best in this regard.

\section{Competing Interests}

The authors declare that there is no conflict of interests regarding the publication of this article.

\section{Acknowledgments}

This study was supported by the Shanghai Science and Technology Commission (10JC1409000 and 15140904100) and the National Natural Science Foundation of China (31371335).

\section{References}

[1] M. Hidalgo, S. Cascinu, J. Kleeff et al., "Addressing the challenges of pancreatic cancer: future directions for improving outcomes," Pancreatology, vol. 15, no. 1, pp. 8-18, 2015.

[2] C. Verbeke, M. Löhr, J. S. Karlsson, and M. Del Chiaro, "Pathology reporting of pancreatic cancer following neoadjuvant therapy: challenges and uncertainties," Cancer Treatment Reviews, vol. 41, no. 1, pp. 17-26, 2015.

[3] C. D. Logsdon and W. Lu, "The significance of ras activity in pancreatic cancer initiation," International Journal of Biological Sciences, vol. 12, no. 3, pp. 338-346, 2016.

[4] A. L. Mihaljevic, C. W. Michalski, H. Friess, and J. Kleeff, "Molecular mechanism of pancreatic cancer-understanding proliferation, invasion, and metastasis," Langenbeck's Archives of Surgery, vol. 395, no. 4, pp. 295-308, 2010.

[5] M. R. Schweiger, M. Kerick, B. Timmermann, and M. Isau, "The power of NGS technologies to delineate the genome organization in cancer: from mutations to structural variations and epigenetic alterations," Cancer and Metastasis Reviews, vol. 30, no. 2, pp. 199-210, 2011.

[6] T. A. Sohn, G. H. Su, B. Ryu, C. J. Yeo, and S. E. Kern, "Highthroughput drug screening of the DPC4 tumor-suppressor pathway in human pancreatic cancer cells," Annals of Surgery, vol. 233, no. 5, pp. 696-703, 2001.

[7] M. Guo, H. Wei, J. Hu, S. Sun, J. Long, and X. Wang, "U0126 inhibits pancreatic cancer progression via the KRAS signaling pathway in a zebrafish xenotransplantation model," Oncology Reports, vol. 34, no. 2, pp. 699-706, 2015.

[8] Y. Q. Tao, X. P. Zhou, C. Z. Liang et al., "TGF- $\beta 3$ and IGF-1 synergy ameliorates nucleus pulposus mesenchymal stem cell differentiation towards the nucleus pulposus cell type through MAPK/ERK signaling," Growth Factors, vol. 33, no. 5-6, pp. 326-336, 2015.

[9] L. Zheng, G. Jiang, H. Mei et al., "Small RNA interferencemediated gene silencing of heparanase abolishes the invasion, metastasis and angiogenesis of gastric cancer cells," BMC Cancer, vol. 10, article 33, 2010.

[10] A. Krühn, A. Wang, J. H. Fruehauf, and H. Lage, "Delivery of short hairpin RNAs by transkingdom RNA interference modulates the classical ABCB1-mediated multidrug-resistant phenotype of cancer cells," Cell Cycle, vol. 8, no. 20, pp. 3349$3354,2009$.

[11] S. Lunardi, R. J. Muschel, and T. B. Brunner, "The stromal compartments in pancreatic cancer: are there any therapeutic targets?" Cancer Letters, vol. 343, no. 2, pp. 147-155, 2014.

[12] R. M. Perera and N. Bardeesy, "Pancreatic cancer metabolism: breaking it down to build it back up," Cancer Discovery, vol. 5, no. 12, pp. 1247-1261, 2015.

[13] A. Grimont, A. V. Pinho, M. J. Cowley et al., "SOX9 regulates ERBB signalling in pancreatic cancer development," Gut, vol. 64, no. 11, pp. 1790-1799, 2015.

[14] M. Javle, Y. Li, D. Tan et al., "Biomarkers of TGF- $\beta$ signaling pathway and prognosis of pancreatic cancer," PLoS ONE, vol. 9, no. 1, Article ID e85942, 2014. 
[15] S.-C. Tang and Y.-C. Chen, "Novel therapeutic targets for pancreatic cancer," World Journal of Gastroenterology, vol. 20, no. 31, pp. 10825-10844, 2014.

[16] G. Vassaux, A. Angelova, P. Baril, P. Midoux, J. Rommelaere, and P. Cordelier, "The promise of gene therapy for pancreatic cancer," Human Gene Therapy, vol. 27, no. 2, pp. 127-133, 2016.

[17] S.-X. Liu, Z.-S. Xia, and Y.-Q. Zhong, "Gene therapy in pancreatic cancer," World Journal of Gastroenterology, vol. 20, no. 37, pp. 13343-13368, 2014.

[18] R. J. Brais, S. E. Davies, M. O’Donovan et al., “Direct histological processing of EUS biopsies enables rapid molecular biomarker analysis for interventional pancreatic cancer trials," Pancreatology, vol. 12, no. 1, pp. 8-15, 2012.

[19] D. Yeo, H. He, G. S. Baldwin, and M. Nikfarjam, "The role of p21activated kinases in pancreatic cancer," Pancreas, vol. 44, no. 3 , pp. 363-369, 2015.

[20] E. K. Colvin and C. J. Scarlett, "A historical perspective of pancreatic cancer mouse models," Seminars in Cell and Developmental Biology, vol. 27, pp. 96-105, 2014.

[21] F. Yuan, Y.-H. Zhang, S. Wan, S. Wang, and X.-Y. Kong, "Mining for candidate genes related to pancreatic cancer using proteinprotein interactions and a shortest path approach," BioMed Research International, vol. 2015, Article ID 623121, 12 pages, 2015.

[22] M. Kanehisa, Y. Sato, M. Kawashima, M. Furumichi, and M. Tanabe, "KEGG as a reference resource for gene and protein annotation," Nucleic Acids Research, vol. 44, no. 1, pp. D457D462, 2016.

[23] M. A. Harris, J. I. Deegan, J. Lomax et al., "The gene ontology project in 2008," Nucleic Acids Research, vol. 36, pp. D440D444, 2008.

[24] H. Peng, F. Long, and C. Ding, "Feature selection based on mutual information: criteria of max-dependency, maxrelevance, and min-redundancy," IEEE Transactions on Pattern Analysis and Machine Intelligence, vol. 27, no. 8, pp. 1226-1238, 2005.

[25] H. Ogata, S. Goto, K. Sato, W. Fujibuchi, H. Bono, and M. Kanehisa, "KEGG: kyoto encyclopedia of genes and genomes," Nucleic Acids Research, vol. 27, no. 1, pp. 29-34, 1999.

[26] A. Franceschini, D. Szklarczyk, S. Frankild et al., "STRING v9.1: protein-protein interaction networks, with increased coverage and integration," Nucleic Acids Research, vol. 41, no. 1, pp. D808D815, 2013.

[27] P. Carmona-Saez, M. Chagoyen, F. Tirado, J. M. Carazo, and A. Pascual-Montano, "GENECODIS: a web-based tool for finding significant concurrent annotations in gene lists," Genome Biology, vol. 8, no. 1, article R3, 2007.

[28] T. Huang, L. Chen, Y.-D. Cai, and K.-C. Chou, "Classification and analysis of regulatory pathways using graph property, biochemical and physicochemical property, and functional property," PLoS ONE, vol. 6, no. 9, Article ID e25297, 2011.

[29] T. Huang, J. Zhang, Z.-P. Xu et al., "Deciphering the effects of gene deletion on yeast longevity using network and machine learning approaches," Biochimie, vol. 94, no. 4, pp. 1017-1025, 2012.

[30] J. Yang, L. Chen, X. Kong, T. Huang, and Y.-D. Cai, "Analysis of tumor suppressor genes based on gene ontology and the KEGG pathway," PLoS ONE, vol. 9, no. 9, Article ID e107202, 2014.

[31] L. Chen, Y. Zhang, M. Zheng, T. Huang, and Y. Cai, "Identification of compound-protein interactions through the analysis of gene ontology, KEGG enrichment for proteins and molecular fragments of compounds," Molecular Genetics and Genomics, vol. 291, no. 6, pp. 2065-2079, 2016.

[32] L. Chen, C. Chu, and K. Feng, "Predicting the types of metabolic pathway of compounds using molecular fragments and sequential minimal optimization," Combinatorial Chemistry \& High Throughput Screening, vol. 19, no. 2, pp. 136-143, 2016.

[33] Y. Zhang, C. Ding, and T. Li, "Gene selection algorithm by combining reliefF and mRMR," BMC Genomics, vol. 9, no. 2, article S27, 2008.

[34] L. Liu, L. Chen, Y.-H. Zhang et al., "Analysis and prediction of drug-drug interaction by minimum redundancy maximum relevance and incremental feature selection," Journal of Biomolecular Structure and Dynamics, 2016.

[35] Z. Li, X. Zhou, Z. Dai, and X. Zou, "Classification of G-protein coupled receptors based on support vector machine with maximum relevance minimum redundancy and genetic algorithm," BMC Bioinformatics, vol. 11, article 325, 2010.

[36] H. Mohabatkar, M. Mohammad Beigi, and A. Esmaeili, "Prediction of GABAA receptor proteins using the concept of Chou's pseudo-amino acid composition and support vector machine," Journal of Theoretical Biology, vol. 281, no. 1, pp. 18-23, 2011.

[37] L. Chen, Y. Zhang, T. Huang, and Y. Cai, "Gene expression profiling gut microbiota in different races of humans," Scientific Reports, vol. 6, article 23075, 2016.

[38] Z. Liu, J. Han, H. Lv, J. Liu, and R. Liu, "Computational identification of circular RNAs based on conformational and thermodynamic properties in the flanking introns," Computational Biology and Chemistry, vol. 61, pp. 221-225, 2016.

[39] L. Chen, C. Chu, J. Lu, X. Kong, T. Huang, and Y.-D. Cai, “Gene ontology and KEGG pathway enrichment analysis of a drug target-based classification system," PLoS ONE, vol. 10, no. 5, Article ID e0126492, 2015.

[40] S. A. Korkmaz, M. F. Korkmaz, M. Poyraz, and F. Yakuphanoglu, "Diagnosis of breast cancer nano-biomechanics images taken from atomic force microscope," Journal of Nanoelectronics and Optoelectronics, vol. 11, no. 4, pp. 551-559, 2016.

[41] L. Chen, C. Chu, T. Huang, X. Kong, and Y.-D. Cai, "Prediction and analysis of cell-penetrating peptides using pseudo-amino acid composition and random forest models," Amino Acids, vol. 47, no. 7, pp. 1485-1493, 2015.

[42] T. Huang, M. Wang, and Y.-D. Cai, "Analysis of the preferences for splice codes across tissues," Protein and Cell, vol. 6, no. 12, pp. 904-907, 2015.

[43] S. Wang, Y. Zhang, J. Lu, W. Cui, J. Hu, and Y. Cai, "Analysis and identification of aptamer-compound interactions with a maximum relevance minimum redundancy and nearest neighbor algorithm," BioMed Research International, vol. 2016, Article ID 8351204, 9 pages, 2016.

[44] T. Huang, Y. Shu, and Y.-D. Cai, "Genetic differences among ethnic groups," BMC Genomics, vol. 16, article 1093, 2015.

[45] L. Chen, C. Chu, Y.-H. Zhang et al., "Analysis of gene expression profiles in the human brain stem, cerebellum and cerebral cortex," PLoS ONE, vol. 11, no. 7, Article ID e0159395, 2016.

[46] J. Han, F. Wang, S.-Q. Yuan et al., "Reduced expression of p21-activated protein kinase 1 correlates with poor histological differentiation in pancreatic cancer," BMC Cancer, vol. 14, no. 1, article 650, 2014.

[47] X. Bai, X. Zhi, Q. Zhang et al., "Inhibition of protein phosphatase $2 \mathrm{~A}$ sensitizes pancreatic cancer to chemotherapy by increasing drug perfusion via HIF- $1 \alpha$-VEGF mediated angiogenesis," Cancer Letters, vol. 355, no. 2, pp. 281-287, 2014. 
[48] N. P. Zimmerman, I. Roy, A. D. Hauser, J. M. Wilson, C. L. Williams, and M. B. Dwinell, "Cyclic AMP regulates the migration and invasion potential of human pancreatic cancer cells," Molecular Carcinogenesis, vol. 54, no. 3, pp. 203-215, 2015.

[49] R. Kang, W. Hou, Q. Zhang et al., "RAGE is essential for oncogenic KRAS-mediated hypoxic signaling in pancreatic cancer," Cell Death and Disease, vol. 5, no. 10, Article ID e1480, 2014.

[50] Q. Wang, Q. Ni, X. Wang, H. Zhu, Z. Wang, and J. Huang, "High expression of RAB27A and TP53 in pancreatic cancer predicts poor survival," Medical Oncology, vol. 32, no. 1, p. 372, 2015.

[51] A. Black, P. F. Pinsky, R. L. Grubb et al., "Sex steroid hormone metabolism in relation to risk of aggressive prostate cancer," Cancer Epidemiology Biomarkers \& Prevention, vol. 23, no. 11, pp. 2374-2382, 2014.

[52] D.-S. Ming, S. Pham, S. Deb et al., "Pomegranate extracts impact the androgen biosynthesis pathways in prostate cancer models in vitro and in vivo," The Journal of Steroid Biochemistry and Molecular Biology, vol. 143, pp. 19-28, 2014.

[53] E. J. Duell, N. Travier, L. Lujan-Barroso et al., "Menstrual and reproductive factors in women, genetic variation in CYP17A1, and pancreatic cancer risk in the European prospective investigation into cancer and nutrition (EPIC) cohort," International Journal of Cancer, vol. 132, no. 9, pp. 2164-2175, 2013.

[54] J. Zhou and Y. Du, "Acquisition of resistance of pancreatic cancer cells to 2-methoxyestradiol is associated with the upregulation of manganese superoxide dismutase," Molecular Cancer Research, vol. 10, no. 6, pp. 768-777, 2012.

[55] J. A. Pierzynski, M. A. Hildebrandt, A. M. Kamat et al., "Genetic variants in the Wnt/ $\beta$-catenin signaling pathway as indicators of bladder cancer risk," The Journal of Urology, vol. 194, no. 6, pp. 1771-1776, 2015.

[56] N. Yoshida, T. Kinugasa, K. Ohshima et al., "Analysis of Wnt and $\beta$-catenin expression in advanced colorectal cancer," Anticancer Research, vol. 35, no. 8, pp. 4403-4410, 2015.

[57] M. Ilmer, A. R. Boiles, I. Regel et al., "RSPO2 enhances canonical wnt signaling to confer stemness-associated traits to susceptible pancreatic cancer cells," Cancer Research, vol. 75, no. 9, pp. 18831896, 2015.

[58] I. Wall and I. G. H. Schmidt-Wolf, "Effect of Wnt inhibitors in pancreatic cancer," Anticancer Research, vol. 34, no. 10, pp. 53755380, 2014.

[59] Y. Zhao, Y. Yang, J. Trovik et al., "A novel Wnt regulatory axis in endometrioid endometrial cancer," Cancer Research, vol. 74, no. 18, pp. 5103-5117, 2014.

[60] Y. Han, Y. Luo, Y. Wang, Y. Chen, M. Li, and Y. Jiang, "Hepatocyte growth factor increases the invasive potential of PC-3 human prostate cancer cells via an ERK/MAPK and ZEB-1 signaling pathway," Oncology Letters, vol. 11, no. 1, pp. 753-759, 2016.

[61] M. Beuran, I. Negoi, S. Paun et al., "The epithelial to mesenchymal transition in pancreatic cancer: a systematic review," Pancreatology, vol. 15, no. 3, pp. 217-225, 2015.

[62] W. Zhou, A. M. Jubb, K. Lyle et al., "PAK1 mediates pancreatic cancer cell migration and resistance to MET inhibition," The Journal of Pathology, vol. 234, no. 4, pp. 502-513, 2014.

[63] M. Quimbaya, E. Raspé, G. Denecker et al., "Deregulation of the replisome factor MCMBP prompts oncogenesis in colorectal carcinomas through chromosomal instability," Neoplasia, vol. 16, no. 9, pp. 694-709, 2014.
[64] Y. Matsuda, T. Ishiwata, N. Izumiyama-Shimomura et al., "Gradual telomere shortening and increasing chromosomal instability among PanIN grades and normal ductal epithelia with and without cancer in the pancreas," PLoS ONE, vol. 10, no. 2, Article ID e0117575, 2015.

[65] W.-Z. Wang, Q.-H. Pu, X.-H. Lin et al., "Silencing of miR21 sensitizes CML CD34+ stem/progenitor cells to imatinibinduced apoptosis by blocking PI3K/AKT pathway," Leukemia Research, vol. 39, no. 10, pp. 1117-1124, 2015.

[66] Y.-T. Zheng, H.-Y. Yang, T. Li et al., "Amiloride sensitizes human pancreatic cancer cells to erlotinib in vitro through inhibition of the PI3K/AKT signaling pathway," Acta Pharmacologica Sinica, vol. 36, no. 5, pp. 614-626, 2015.

[67] N. Walsh, A. Larkin, N. Swan et al., "RNAi knockdown of Hop (Hsp70/Hsp90 organising protein) decreases invasion via MMP-2 down regulation," Cancer Letters, vol. 306, no. 2, pp. 180-189, 2011.

[68] Y. I. Chang, X. You, G. Kong et al., "Loss of Dnmt3a and endogenous $\mathrm{Kras}(\mathrm{G} 12 \mathrm{D}+)$ cooperate to regulate hematopoietic stem and progenitor cell functions in leukemogenesis," Leukemia, vol. 29, pp. 1847-1856, 2015.

[69] C. Oancea, B. Rüster, B. Brill et al., "STAT activation status differentiates leukemogenic from nonleukemogenic stem cells in AML and is suppressed by arsenic in $\mathrm{t}(6 ; 9)$-positive AML," Genes and Cancer, vol. 5, no. 11-12, pp. 378-392, 2014.

[70] M. A. Macha, S. Rachagani, S. Gupta et al., "Guggulsterone decreases proliferation and metastatic behavior of pancreatic cancer cells by modulating JAK/STAT and Src/FAK signaling," Cancer Letters, vol. 341, no. 2, pp. 166-177, 2013.

[71] Y. Bian, Y. Yu, S. Wang, and L. Li, "Up-regulation of fatty acid synthase induced by EGFR/ERK activation promotes tumor growth in pancreatic cancer," Biochemical and Biophysical Research Communications, vol. 463, no. 4, pp. 612-617, 2015.

[72] J. Liu, D. Xu, Q. Wang, D. Zheng, X. Jiang, and L. Xu, "LPS induced miR-181a promotes pancreatic cancer cell migration via targeting PTEN and MAP2K4," Digestive Diseases and Sciences, vol. 59, no. 7, pp. 1452-1460, 2014.

[73] D. C. Morran, J. M. Wu, N. B. Jamieson et al., "Targeting mTOR dependency in pancreatic cancer," Gut, vol. 63, no. 9, pp. 14811489, 2014.

[74] V. Nair, S. Sreevalsan, R. Basha et al., "Mechanism of metformin-dependent inhibition of mammalian target of rapamycin (mTOR) and Ras activity in pancreatic cancer: role of specificity protein (Sp) transcription factors," The Journal of Biological Chemistry, vol. 289, no. 40, pp. 27692-27701, 2014.

[75] F. Wei, Y. Zhang, L. Geng, P. Zhang, G. Wang, and Y. Liu, "mTOR inhibition induces EGFR feedback activation in association with its resistance to human pancreatic cancer," International Journal of Molecular Sciences, vol. 16, no. 2, pp. 3267-3282, 2015.

[76] F. Wang, H. Li, X.-G. Yan et al., "Alisertib induces cell cycle arrest and autophagy and suppresses epithelial-to-mesenchymal transition involving PI3K/Akt/mTOR and sirtuin 1mediated signaling pathways in human pancreatic cancer cells," Drug Design, Development and Therapy, vol. 9, pp. 575-601, 2015.

[77] R. Guo, Y. Wang, W.-Y. Shi, B. Liu, S.-Q. Hou, and L. Liu, "MicroRNA miR-491-5p targeting both TP53 and Bcl-XL induces cell apoptosis in SW1990 pancreatic cancer cells through mitochondria mediated pathway," Molecules, vol. 17, no. 12, pp. 14733-14747, 2012. 
[78] C. Fiorini, M. Menegazzi, C. Padroni et al., "Autophagy induced by p53-reactivating molecules protects pancreatic cancer cells from apoptosis," Apoptosis, vol. 18, no. 3, pp. 337-346, 2013.

[79] F. Wei, Y. D. Zhang, L. Geng, P. Zhang, G. Y. Wang, and Y. Liu, "mTOR inhibition induces EGFR feedback activation in association with its resistance to human pancreatic cancer," International Journal of Molecular Sciences, vol. 16, no. 2, pp. 3267-3282, 2015.

[80] K. Taniuchi, M. Furihata, K. Hanazaki et al., "Peroxiredoxin 1 promotes pancreatic cancer cell invasion by modulating p38 MAPK activity," Pancreas, vol. 44, no. 2, pp. 331-340, 2015.

[81] R. Subramani, R. Lopez-Valdez, A. Arumugam, S. Nandy, T. Boopalan, and R. Lakshmanaswamy, "Targeting insulin-like growth factor 1 receptor inhibits pancreatic cancer growth and metastasis," PLoS ONE, vol. 9, no. 5, Article ID e97016, 2014.

[82] G. Androutsopoulos, G. Adonakis, A. Liava, P. Ravazoula, and G. Decavalas, "Expression and potential role of ErbB receptors in type II endometrial cancer," European Journal of Obstetrics Gynecology and Reproductive Biology, vol. 168, no. 2, pp. 204208, 2013.

[83] A. Grimont, A. V. Pinho, M. J. Cowley et al., "SOX9 regulates ERBB signalling in pancreatic cancer development," Gut, vol. 64, no. 11, pp. 1790-1799, 2015.

[84] F. C. Bronfman, O. M. Lazo, C. Flores, and C. A. Escudero, "Spatiotemporal intracellular dynamics of neurotrophin and its receptors. Implications for neurotrophin signaling and neuronal function," in Neurotrophic Factors, vol. 220 of Handbook of Experimental Pharmacology, pp. 33-65, 2014.

[85] P. A. Forsyth, N. Krishna, S. Lawn et al., "p75 neurotrophin receptor cleavage by $\alpha$ - and $\gamma$-secretases is required for neurotrophin-mediated proliferation of brain tumor-initiating cells," Journal of Biological Chemistry, vol. 289, no. 12, pp. 80678085, 2014.

[86] S. Lawn, N. Krishna, A. Pisklakova et al., "Neurotrophin signaling via TrkB and TrkC receptors promotes the growth of brain tumor-initiating cells," The Journal of Biological Chemistry, vol. 290, no. 6, pp. 3814-3824, 2015.

[87] G. Esposito, E. Capoccia, F. Turco et al., "Palmitoylethanolamide improves colon inflammation through an enteric glia/toll like receptor 4-dependent PPAR- $\alpha$ activation," Gut, vol. 63, no. 8, pp. 1300-1312, 2014.

[88] S. J. Miknyoczki, A. J. P. Klein-Szanto, and B. A. Ruggeri, "Neurotrophin-Trk receptor interactions in neoplasia: a possible role in interstitial and perineural invasion in ductal pancreatic cancer," Critical Reviews in Oncogenesis, vol. 7, no. 1-2, pp. 89-100, 1996.

[89] P. L. Che, Y. F. Yang, X. S. Han et al., "S100A4 promotes pancreatic cancer progression through a dual signaling pathway mediated by Src and focal adhesion kinase," Scientific Reports, vol. 5, article 8453, 2015.

[90] A. B. Frey, "Suppression of T cell responses in the tumor microenvironment," Vaccine, vol. 33, no. 51, pp. 7393-7400, 2015.

[91] D. S. Shin and A. Ribas, "The evolution of checkpoint blockade as a cancer therapy: what's here, what's next?" Current Opinion in Immunology, vol. 33, pp. 23-35, 2015.

[92] J. A. Wallace, F. Li, G. Leone, and M. C. Ostrowski, "Pten in the breast tumor microenvironment: modeling tumor-stroma coevolution," Cancer Research, vol. 71, no. 4, pp. 1203-1207, 2011.

[93] R. A. Weinberg, "Coevolution in the tumor microenvironment," Nature Genetics, vol. 40, no. 5, pp. 494-495, 2008.
[94] J.-H. Xu, J.-J. Fu, X.-L. Wang, J.-Y. Zhu, X.-H. Ye, and S.-D. Chen, "Hepatitis B or C viral infection and risk of pancreatic cancer: a meta-analysis of observational studies," World Journal of Gastroenterology, vol. 19, no. 26, pp. 4234-4241, 2013.

[95] A. Kabir, "Comment on: risk of pancreatic cancer in relation to $\mathrm{ABO}$ blood group and hepatitis $\mathrm{C}$ virus infection in korea: $\mathrm{A}$ Case-Control Study," Journal of Korean Medical Science, vol. 28, no. 7, pp. 1114-1115, 2013.

[96] S. J. Miknyoczki, W. Wan, H. Chang et al., “The neurotrophintrk receptor axes are critical for the growth and progression of human prostatic carcinoma and pancreatic ductal adenocarcinoma xenografts in nude mice," Clinical Cancer Research, vol. 8, no. 6, pp. 1924-1931, 2002.

[97] J. Guillermet-Guibert, L. Davenne, D. Pchejetski et al., "Targeting the sphingolipid metabolism to defeat pancreatic cancer cell resistance to the chemotherapeutic gemcitabine drug," Molecular Cancer Therapeutics, vol. 8, no. 4, pp. 809-820, 2009.

[98] J. Gillespie, J. F. Dye, M. Schachter, and P. J. Guillou, "Inhibition of pancreatic cancer cell growth in vitro by the tyrphostin group of tyrosine kinase inhibitors," British Journal of Cancer, vol. 68, no. 6, pp. 1122-1126, 1993.

[99] S. Goel, J. Huang, and L. Klampfer, "K-Ras, Intestinal homeostasis and colon cancer," Current Clinical Pharmacology, vol. 10, no. 1, pp. 73-81, 2015. 

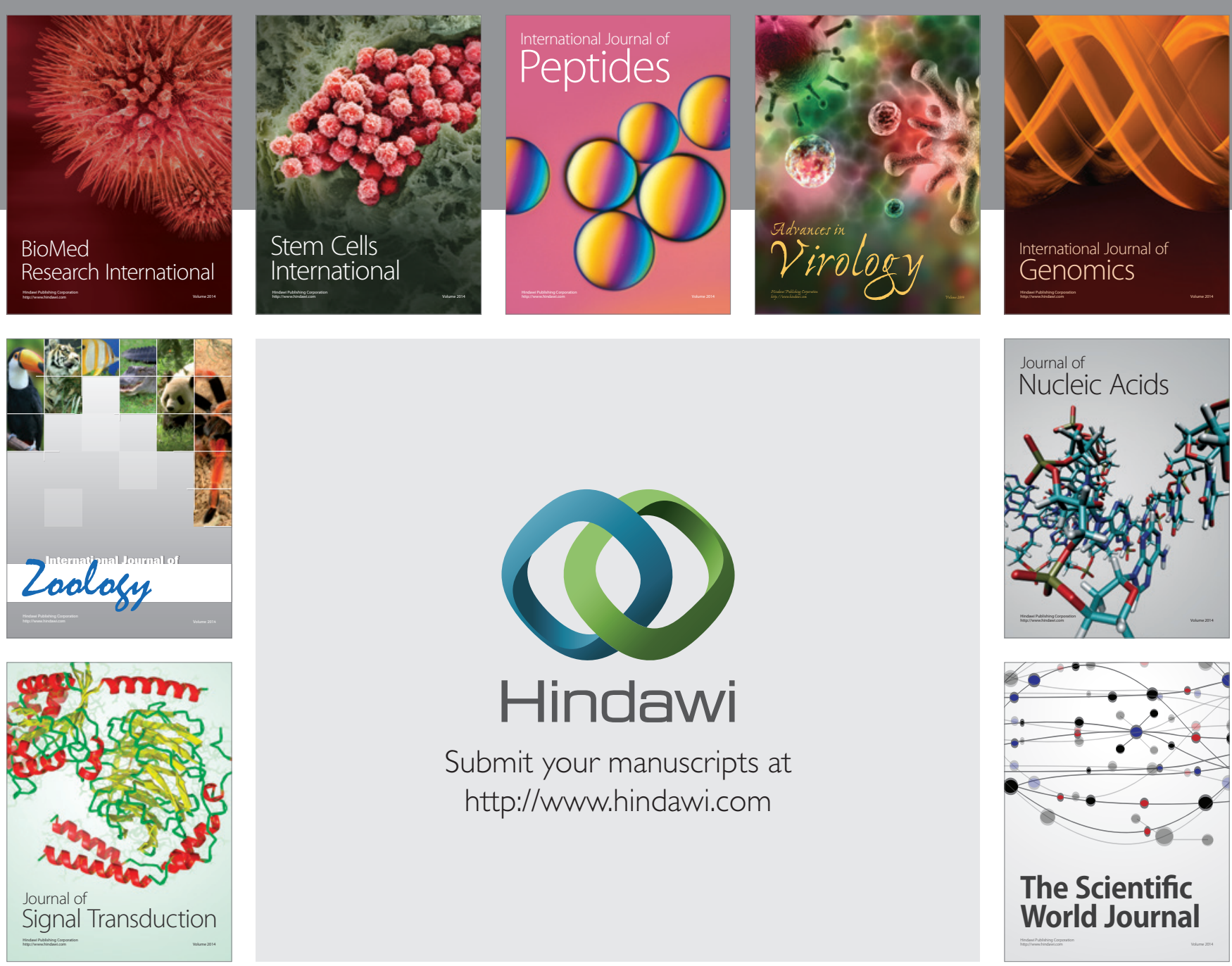

Submit your manuscripts at

http://www.hindawi.com
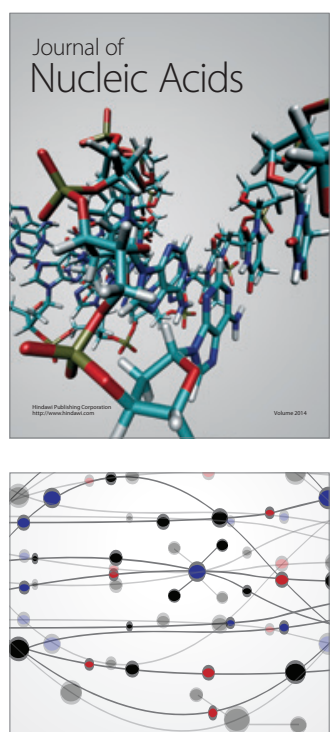

The Scientific World Journal
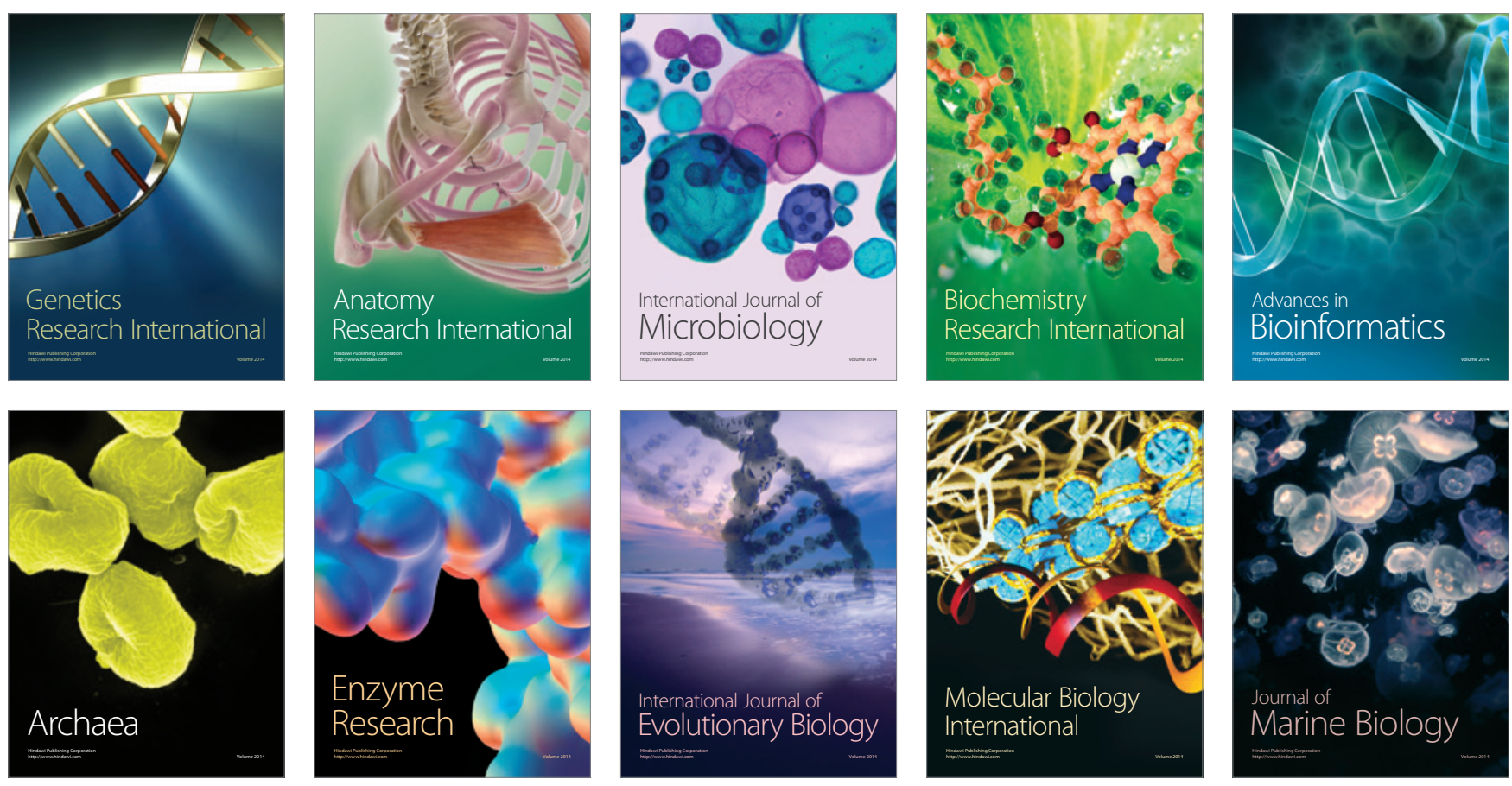